\title{
The clinical and radiological profile of primary lateral sclerosis: an annotation on its pathological and clinical background
}

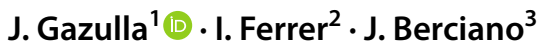

Received: 4 November 2019 / Revised: 23 November 2019 / Accepted: 25 November 2019 / Published online: 5 December 2019

c) Springer-Verlag GmbH Germany, part of Springer Nature 2019

\section{Dear Sirs,}

We read with interest the article by Finegan et al. on primary lateral sclerosis (PLS) [1], highlighting abnormalities found in the cerebellum and cerebral white matter, and atrophy of the body of corpus callosum, as detected by MRI. The authors expressed concern about the scarcity of pathological examinations in PLS that could confirm their findings, and proposed that cerebellar signs should be looked for in cases of PLS.

Recently, we had the chance to study a kindred with PLS [2], a much rarer variant than the sporadic one. On neuropathological examination in one case, macroscopic atrophy of the frontal lobes due to severely reduced white matter, and of the body of the corpus callosum, was found in one case, as well as marked reduction of myelin and nerve fibres on microscopical examination. Corticospinal tract degeneration was found in another case, together with depletion of Purkinje cells in the cerebellum, and of neurons in the olivary nuclei. MRI revealed atrophy of the body of the corpus callosum in two cases, and brain subcortical hyperintensities, in one.

On clinical grounds, inaugural cerebellar ataxia was detected in two patients, superseded later by generalized spasticity; non-fluent aphasia was found in every case.

Hence, we had demonstrated atrophy of the corpus callosum, cerebello-olivary atrophy and cerebral white matter

\section{J. Gazulla}

josegazulla@gmail.com

1 Department of Neurology, Hospital Universitario Miguel Servet, Zaragoza, Spain

2 Department of Pathology and Experimental Therapeutics, University of Barcelona, IDIBELL-Bellvitge University Hospital, CIBERNED, Hospitalet de Llobregat, Barcelona, Spain

3 Department of Neurology, Hospital UniversitarioMarqués de Valdecilla (IDIVAL), University of Cantabria, CIBERNED, Santander, Spain abnormalities, and found cerebellar ataxia, in PLS. Our neuropathological and clinical findings provide an answer to the proposals of Finegan et al. based solely on MRI findings.

As PLS constitutes a clinical diagnosis at present, due to a lack of diagnostic markers, considerable potential variability in its manifestations is to be expected, as found in our study. Further research is needed to fully elucidate the spectrum of PLS.

\section{Compliance with ethical standards}

Conflicts of interest None to be declared.

Ethical approval This article does not contain any studies with human participants or animals performed by any of the authors.

\section{Reference}

1. Finegan E, Chipika RH, Shing SL, Doherty MA, Hengeveld JC, Vajda A, Donaghy C, McLaughlin RL, Pender N, Hardiman O, Bede $\mathrm{P}$ (2019) The clinical and radiological profile of primary lateral sclerosis: a population-based study. J Neurol 266:2718-2733

2. Gazulla J, Ferrer I, Izquierdo-Alvarez S, Alvarez S, Sánchez-Alcudia R, Bestué-Cardiel M, Seral M, Benavente I, Sierra-Martínez E, Berciano J (2019) Hereditary primary lateral sclerosis and progressive nonfluent aphasia. J Neurol 266:1079-1090 